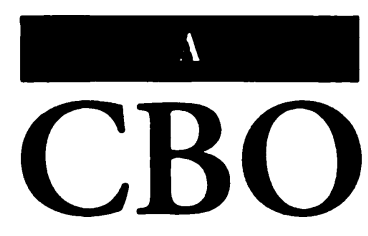

S I I I I

\title{
The Budget and Economic Outlook: An Update
}

September 2004

\author{
A Report to the Senate and \\ House Committees on the Budget
}

The Congress of the United States $₫$ Congressional Budget Office 